\title{
Special Types of Partitioned Matrices
}

\author{
Emilie V. Haynsworth*
}

(September 14, 1960)

\begin{abstract}
This paper extends the results of two previous papers on partitioned matrices. General reduction formulas are given for partitioned matrices $A$ of order $n p$ satisfying $A\left(X \times I_{p}\right)$ $=\left(X \times I_{p}\right) B$, where $B$ is a matrix of order $r p$ and $X \times I_{p}$ represents the direct product of an $n \times r$ matrix $X$ of rank $r$ with the identity matrix of order $p$. These formulas are related to the formulas given in the previous papers for partitioned matrices satisfying $A\left(I_{p} \times X\right)$ $=\left(I_{p} \times X\right) B$.
\end{abstract}

\section{Introduction}

In a recent paper L. S. Goddard and H. Schneider [4] ${ }^{1}$ discussed the relationship between matrices $A$ and $B$, of orders $n$ and $m$ respectively, which satisfy

$$
A X=X B
$$

for some $n \times m$ matrix, $X$, of rank $r>0$.

The results of Goddard and Schneider were generalized in a paper by the author [5] for partitioned matrices $A$ and $B$, in which corresponding submatrices (or blocks), $A_{i j}$ and $B_{i j}$, satisfy an analogous relationship to that given by (1); i.e.,

$$
A_{i j} X_{j}=X_{i} B_{i j}, \quad(i, j=1, \ldots, p)
$$

where $A_{i j}$ is $n_{i} \times n_{j}, X_{i}$ is $n_{i} \times m_{i}$ of rank $r_{i}$, and $B_{i j}$ is $m_{i} \times m_{j}$.

If, in (2), each $A_{i j}$ is square, of order $n, B_{i j}$ is square of order $r$, and all $X_{i}$ are equal to an $n \times r$ matrix, $X$, of rank $r$, then $A$ is of order $p n, B$ of order $p r$, and (2) can be written

$$
A\left(I_{p} \times X\right)=\left(I_{p} \times X\right) B
$$

where $\left(I_{p} \times X\right)$ is a $p n \times p r$ matrix which is the direct product of the identity matrix of order $p$ with the matrix $X$; i.e.,

$$
I_{p} \times X=\left(\begin{array}{lllll}
X & & & & \\
& X & & & \\
& & \cdot & & \\
& & \cdot & & \\
& & & \cdot & \\
& & & & X
\end{array}\right)
$$

*Present address: Auburn University, Auburn, Ala.

1 Figures in brackets indicate the literature references at the end of this paper.
(The general definition for direct products is given in section 3.)

It was shown by the author in [6] that, given a matrix $A$ satisfying (3) for some matrices $B$ and $X$, there exists a matrix $P$, which can be constructed from $X$, such that

$$
P^{-1} A P=\left(\begin{array}{ll}
B & * \\
0 & C
\end{array}\right),
$$

so that $p r$ of the roots of $A$ are the roots of $B$. Thus, if $r<n$, or of $B$ is reduced, (4) gives a reduction formula for $A$. Naturally a matrix satisfying (1) satisifes (3) with $p=1$.

In theorem 2 of this paper a similar reduction formula to those for matrices satisfying (1) and (3) is given for partitioned matrices $A$ of order $p n$ satisfying

$$
A\left(X \times I_{p}\right)=\left(X \times I_{p}\right) B
$$

for some $n \times r$ matrix $X$ of rank $r$, and the corresponding partitioned matrix $B$ of order $p r$. The relationship between matrices satisfying (3) and (5) is discussed more fully in the next section.

Other reduction formulas for partitioned matrices are contained in the results of B. Friedman [3], S. N. Afriat [1], and J. Williamson [10]. These formulas will be shown to be special cases of theorem 2.

The reduction formulas given in section 5 are applied in section 7 to certain special partitioned matrices. In particular, a $9 \times 9$ matrix, involving an arbitrary parameter $\beta$, which arose in a problem on lattice points at the Bureau of Standards, is reduced by these formulas to a set of matrices of order $4,2,2$, and 1 and from these reduced matrices all eigenvalues are found as functions of $\beta$. 


\section{Inner- and Outer-Related Partitioned Matrices}

The formulas (1), (3), and (5) may be related bv the matrix $X$. For instance, if the matrix $A$ in (1) is a circulant matrix,

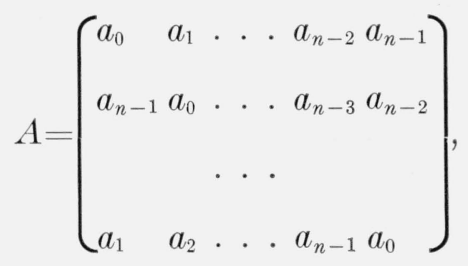

and $X$ is the matrix of its characteristic vectors, then the related matrix satisfying (3) will have each of its submatrices a circulant of order $n$, and the related matrix satisfying (5) will be in circulant form; i.e.,

$$
A=\left(\begin{array}{cccc}
A_{0} & A_{1} & \ldots & A_{n-1} \\
A_{n-1} & A_{0} & \ldots & A_{n-2} \\
& \ldots & \\
A_{1} & A_{2} & \ldots & A_{0}
\end{array}\right) .
$$

A matrix satisfying both (3) and (5) would be one which has the form (7), where each submatrix $A_{i}$ has the form (6). D. E. Rutherford [9] has given reduction formulas for matrices of this type as well as for matrices related to tridiagonal matrices.

We will call matrices satisfying (3) "inner-related" partitioned matrices, or i.r.p. matrices, those satisfying (5) "outer-related," or o.r.p. matrices, and matrices satisfying both conditions "doubly-related," or d.r.p. matrices.

We shall show that dual reduction formulas exist for o.r.p. and i.r.p. matrices and indeed that an o.r.p. matrix can be put into i.r.p. form by a simultaneous permutation of rows and columns.

Since the formulas (3) and (5) are stated in terms of direct products, the general deiinition and three of the properties of direct products are given in section 3 .

\section{Direct Products of Matrices}

W. E. Roth [8] derives some interesting results on direct product matrices and gives the general definition for rectangular matrices, together with some of the properties. The three properties needed for our results are given after the definition below. Most of the properties of direct products can also be found in MacDuffee [7], although he deals only with square matrices.
The direct product of an $m \times n$ matrix, $A$, and a $p \times q$ matrix, $B$, in that order, is an $m p \times n q$ matrix deiined as follows:

$$
A \times B=\left(\begin{array}{cccc}
a_{11} B & a_{12} B & \ldots & a_{1 n} B \\
a_{21} B & a_{22} B & \ldots & a_{2 n} B \\
& & \ldots & \\
& & \ldots & a_{m n} B
\end{array}\right) .
$$

Three properties of direct products:

1. If the products $A C$ and $B D$ exist,

$$
(A \times B)(C \times D)=A C \times B D .
$$

2. If $A$ and $B$ are nonsingular,

$$
(A \times B)^{-1}=\left(A^{-1} \times B^{-1}\right) \text {. }
$$

3. If $A$ is an $m \times n$ matrix and $B$ is a $p \times q$ matrix, $A \times B=P(B \times A) Q$, where $P$ and $Q$ are obtained from the identity matrices $I_{m p}$ and $I_{n q}$ by simultaneous permutation of rows and columns.

\section{Similarity Between O.R.P. and I.R.P. Matrices}

Theorem 1: If $A$ is an outer-related partitioned matrix of order pn, with square blocks of order $p$, satisfying (5), then $A$ can be transformed by simultaneous permutation of rows and columns into an inner-related partitioned matrix, $\tilde{A}$, with blocks of order $n$, satisfying (3).

Proof. Suppose $A$ satisies (5). Using property 3 of direct products, given above, there exist nonsingular permutation matrices, $P$ and $Q$, such that

$$
X \times I_{p}=P\left(I_{p} \times X\right) Q .
$$

Then, from (8) and (5),

$$
A\left[P\left(I_{p} \times X\right) Q\right]=\left[P\left(I_{p} \times X\right) Q\right] B,
$$

or

$$
\left(P^{-1} A P\right)\left(I_{p} \times X\right)=\left(I_{p} \times X\right)\left(Q B Q^{-1}\right) .
$$

So, if we let

$$
\tilde{A}=P^{-1} A P, \quad \tilde{B}=Q B Q^{-1},
$$

the relationship (3) holds for $\tilde{A}$ and $\tilde{B}$, and, since $P$ is a permutation matrix, $\widetilde{A}$ is obtained from $A$ by simultaneous permutation of rows and columns.

From this theorem we can reduce any o.r.p. matrix, if we know the reduction formula for the i.r.p. matrix, by first permuting the rows and columns and then using the i.r.p. reduction formula. However, it is usually much simpler to use the direct reduction formula for o.r.p. matrices which is given in the next section. 


\section{A General Reduction Formula}

Theorem 2: Suppose we have a matrix $A$ of order $n$ satisfying (1), or a matrix of order pn satisfying (3) or (5) for some corresponding matrix $B$, where $X$ is a given $n \times r$ matrix of rank $r$. Then in each case there exists a matrix $P$, depending upon $X$, such that (4) holds, so that $B$ contains $r$ (or pr) of the roots of $A$.

The only new result here is the formula for a matrix satisfying (5), as the formula for a matrix satisfying (1) was given by Goddard and Schneider [4], and the one for a matrix satisfying (3) was given by the author in [6]. The three transformation matrices are given below, however, in order to show the relations among them, but the proof is not given in detail as it appears in the other papers.

Proof. Since $X$ is an $n \times r$ matrix of rank $r$, we will assume

$$
X=\left(\begin{array}{l}
X_{1} \\
X_{2}
\end{array}\right),
$$

where $X_{1}$ is a nonsingular $(r \times r)$ matrix, for if a permutation of the rows of $X$ is necessary to achieve this, it can be matched by a simultaneous permutation of the rows and columns of $A$; i.e., a permutation of the rows of $X$ is equivalent to left multiplication by a nonsingular matrix $Q$, but a matrix $A$ which satisifes (1) also satisities

$$
Q A Q^{-1}(Q X)=(Q X) B
$$

In each case below we will use the matrix $R$, which we construct as follows: The first $r$ columns of $R$ are those of the $n \times r$ matrix $X$, which we assume to have the form (9). The remaining $n-r$ columns consist of an $r \times(n-r)$ block of zeros and the identity matrix of order $n-r$, i.e.

$$
R=\left(\begin{array}{cc}
X_{1} & 0 \\
X_{2} & I_{n-r}
\end{array}\right) .
$$

Then

$$
R^{-1}=\left(\begin{array}{cc}
X_{1}^{-1} & 0 \\
-X_{2} X_{1}^{-1} & I_{n-\tau}
\end{array}\right) .
$$

Using $R$ we can construct the transformation matrices for nonpartitioned matrices satisfying (1), or partitioned matrices satisfying (3) or (5) or both.

\section{Case 1. Non-partitioned matrices}

Suppose $A$ is an $n \times n$ matrix satisfying (1) where $X$ is an $n \times r$ matrix of rank $r$ and $B$ is an $r \times r$ matrix. It was shown by the author in [6] that (4) holds for the matrix $A$ if we let $P=R$.

\section{Case 2. I.R.P. matrices}

If $A$ is a partitioned matrix of order $n p$, with blocks of order $n$, satisfying (3), $A$ also satisfies (1) where the matrix $I_{p} \times X$ replaces the matrix $X$. In this case it was shown by the author in [6] that, if we let $P=I_{p} \times R$, then

$$
P^{-1} A P=\tilde{A},
$$

where $\tilde{A}$ can be put into the reduced form on the right side of (4) by a simultaneous permutation of rows and columns. In this case $B$ is of order $p r$.

\section{Case 3. O.R.P. matrices}

If $A$ is a partitioned matrix of order $n p$, with blocks of order $p$, satisfying (5), then $A$ also satisfies (1) with $X$ replaced by

$$
\left(X \times I_{p}\right)=\left(\begin{array}{c}
X_{1} \times I_{p} \\
X_{2} \times I_{p}
\end{array}\right),
$$

where, by property 2 of direct products, since $X_{1}$ is nonsingular, $X_{1} \times I_{p}$ is nonsingular. Thus, if we let

$$
P=R \times I_{p}=\left(\begin{array}{ll}
X_{1} \times I_{p} & 0 \\
X_{2} \times I_{p} & I_{n-r} \times I_{p}
\end{array}\right),
$$

(4) will hold in this case also, and $B$ will again be of order $p r$.

\section{Case 4. D.R.P. matrices}

If $A$ is a matrix of order $n p$, with blocks of order $n$, and satisines both (3) and (5), i.e.

and

$$
A\left(X \times I_{n}\right)=\left(X \times I_{n}\right) B,
$$

$$
A\left(I_{p} \times Y\right)=\left(I_{p} \times Y\right) C,
$$

where $X$ is $p \times r$ and $Y$ is $n \times s$, then $A$ can be reduced twice. For, by case 3 , if we let $P_{1}=R_{1} \times I_{n}$, where $R_{1}$ is the $p \times p$ matrix given in case 3 , we have

$$
P_{1}^{-1} A P_{1}=\left(\begin{array}{ll}
B & * \\
0 & D
\end{array}\right),
$$

where $B$ is of order $n r$ and $D$ is of order $n(p-r)$.

It is clear that premultiplication and/or postmultiplication of a partitioned matrix $A$, with blocks of order $n$, by a matrix whose blocks are scalar multiples of the identity matrix of order $n$ produces a matrix whose blocks are linear combinations of the blocks of $A$.

Also, if any set of matrices has the property that

then

$$
A_{i} X=X B_{i}, \quad(i=1, \ldots, t)
$$

$$
\sum\left(c_{i} A_{i} X\right)=\sum\left(c_{i} X B_{i}\right),
$$

or

$$
\left(\sum c_{i} A_{i}\right) X=X\left(\sum c_{i} B_{i}\right),
$$

so that any linear combination of the set satisfies an equation of the form (1) with the same matrix $X$. 
Hence the matrices $B$ and $D$ satisfy

$$
\begin{gathered}
B\left(I_{r} \times Y\right)=\left(I_{r} \times Y\right) E, \\
D\left(I_{p-r} \times Y\right)=\left(I_{p-r} \times Y\right) G,
\end{gathered}
$$

where $E$ is of order $r s$ and $G$ is of order $s(p-r)$. Then, by case 2 , there exist matrices $P_{B}$ and $P_{D}$ such that

$$
\begin{aligned}
& P_{B}^{-1} B P_{B}=\left(\begin{array}{ll}
E & * \\
0 & F
\end{array}\right), \\
& P_{D}^{-1} D P_{D}=\left(\begin{array}{ll}
G & * \\
0 & H
\end{array}\right) .
\end{aligned}
$$

Thus, the roots of $A$ are those of the matrices $E, F$, $G$, and $H$, of order $r s, r(n-s), s(p-r)$, and $(n-s)$ $\cdot(p-r)$, respectively.

It would be easier in this case however, to multiply the two transformation matrices $R_{1} \times I_{n}$ and $I_{p} \times R_{2}$, where $R_{2}$ is the $n \times n$ transformation matrix corresponding to the matrix in case 2 , and obtain, by property 1 of direct products, the single transformation matrix, $P=R_{1} \times R_{2}$, which could be used to put $A$ into the reduced form,

$$
P^{-1} A P=\left[\begin{array}{llll}
E & * & * & * \\
0 & F & * & * \\
0 & 0 & G & * \\
0 & 0 & 0 & H
\end{array}\right) .
$$

For example suppose

$$
S=\left(\begin{array}{ll}
A & B \\
C & D
\end{array}\right)
$$

where $A, B, C$, and $D$ are $3 \times 3$ stochastic matrices, i.e.

$$
\begin{aligned}
& \sum_{j=1}^{3} a_{i j}=a \\
& \sum_{j=1}^{3} b_{i j}=b \\
& \sum_{j=1}^{3} c_{i j}=c, \\
& \sum_{j=1}^{3} d_{i j}=d, \quad(i=1,2,3)
\end{aligned}
$$

then $S$ is an i.r.p. stochastic matrix as discussed in reference [6].

If we have also

$$
A+B=C+D
$$

then $S$ is a d.r.p. stochastic matrix.
Since a stochastic matrix with row-sum $s$ has one root $s$ with corresponding eigenvector $(1,1, \ldots, 1)$ (cf. A. Brauer, [2]), the matrices $R_{1}$ and $R_{2}$ would be

$$
R_{1}=\left(\begin{array}{ll}
1 & 0 \\
1 & 1
\end{array}\right), \quad R_{2}=\left(\begin{array}{lll}
1 & 0 & 0 \\
1 & 1 & 0 \\
1 & 0 & 1
\end{array}\right) .
$$

Then

$$
P=R_{1} \times R_{2}=\left(\begin{array}{ll}
R_{2} & 0 \\
R_{2} & R_{2}
\end{array}\right),
$$

and

$$
P^{-1}=R_{1}^{-1} \times R_{2}^{-1}=\left(\begin{array}{cc}
R_{2}^{-1} & 0 \\
-R_{2}^{-1} & R_{2}^{-1}
\end{array}\right) \text {. }
$$

So

$$
\begin{aligned}
P^{-1} S P & =\left(\begin{array}{cccc}
R_{2}^{-1}(A+B) R_{2} & R_{2}^{-1} B R_{2} \\
0 & & R_{2}^{-1}(D-B) R_{2}
\end{array}\right) \\
& =\left(\begin{array}{cccc}
a+b & * & b & * \\
0 & A_{2}+B_{2} & 0 & B_{2} \\
0 & 0 & d-b & * \\
0 & 0 & 0 & D_{2}-B_{2}
\end{array}\right),
\end{aligned}
$$

where

$$
A_{2}=\left(\begin{array}{ll}
a_{22}-a_{12} & a_{23}-a_{13} \\
a_{32}-a_{12} & a_{33}-a_{13}
\end{array}\right),
$$

and $B_{2}$ and $D_{2}$ have similar forms.

Another example using i.r.p. and o.r.p. transformation matrices is given in section 7 .

In each of the above cases, if $r=n$, the matrix $P$ is much simpler; i.e., for $1, P=X$, for $2, P=I_{P} \times X$, for $3, P=X \times I_{p}$ and for 4 if $s=p, P=X \times Y$.

\section{Theorems by Williamson, Afriat, and Friedman}

It was shown by the author in [6] that Williamson's theorem [10] on partitioned matrices follows from theorem 2, part 2, (theorem 1 in [6]), where $r=n$ and the matrices $B_{i j}$ are triangular.

S. N. Afriat [1] gives a general discussion with a number of theorems on the determinant and characteristic roots of partitioned matrices, $A=\left(A_{i j}\right)$, in which the submatrices $A_{i j}$ all commute. One of his results is a generalization of Williamson's theorem and shows that if the roots of the commutative matrices $A_{i j}$ are $\lambda_{i j}^{(k)}(k=1, \ldots, n ; i, j=1, \ldots, p)$ then the roots of the partitioned matrix $A=\left(A_{i j}\right)$ are roots of the matrices $\lambda_{k}=\left(\lambda_{i j}^{(k)}\right)$. Since, as Afriat proves, commutative matrices can be simultaneously 
reduced to triangular form by the same unitary transformation, it is clear that $A=\left(A_{i j}\right)$ satisfies (3), where $X$ is the unitary matrix in question, and the corresponding matrices, $B_{i j}$, are triangular of order $n$. So Afriat's result on the characteristic roots of $A$ also follows from theorem 2 .

Theorem 2 also provides a new proof for the following theorems by Friedman [3].

Theorem I. Let $A_{1}, A_{2}, \ldots, A_{t}$ be $n$-dimensional square matrices and $B_{1}, B_{2}, \ldots ., B_{t}$ be $p$-dimensional square matrices. Suppose that $A_{1}, A_{2}, \ldots$. . A have a common eigenvector, $\zeta$, and the corresponding eigenvalues are $\lambda_{1}, \lambda_{2}, \ldots, \lambda_{i}$ respectively. Then $p$ of the eigenvalues of

$$
C=B_{1} \times A_{1}+B_{2} \times A_{2}+\ldots+B_{t} \times A_{t}
$$

will be eigervalues of the matrix

$$
D=\lambda_{1} B_{1}+\lambda_{2} B_{2}+\ldots+\lambda_{t} B_{t} .
$$

As Friedman points out, this is a generalization of the well-known theorem that the eigenvalues of $B \times A$ are the products, $\lambda_{i} \mu_{j}(i=1, \ldots, n ; j=1$, . . . p), where $\lambda_{i}$ are the roots of $A$ and $\mu_{j}$ are the roots of $B$.

TheOrem II. Let $C$ be the matrix considered in theorem 1. Suppose the ring generated by the matrices $A_{1}, \ldots, A_{t}$ has an $r$-dimensional representation $(r<n)$ in which the matrix $A_{k}$ is represented by $M_{k}$. Then $p r$ of the eigenvalues of $C$ are eigenvalues of the matrix

$$
D=B_{1} \times M_{1}+B_{2} \times M_{2}+\ldots+B_{t} \times M_{t} .
$$

Since theorem II contains theorem I as a special case with $\tau=1$, we will only prove theorem II.

Proof. Friedman shows in his proof that the statement, " $A_{k}$ is represented by $M_{k}$," is equivalent to

$$
A_{k} X=X M_{k}, \quad(k=1, \ldots, t)
$$

where the columns of the matrix $X$ are the basis vectors for the subspace.

Let the elements of the matrix $B_{k}$ be $b_{i j}^{(k)}(i, j=1$, .., $p ; k=1, \ldots, t)$.

Then from (14), $\dot{C}$ is a partitioned matrix of order $p n$ with blocks of order $n$,

$$
C_{i j}=\sum_{k=1}^{t} b_{i j}^{(k)} A_{k} . \quad(i, j=1, \ldots, p) .
$$

From (16)

$$
\begin{aligned}
& \sum_{k=1}^{t} b_{i j}^{(k)}\left(A_{k} X\right)=\sum_{k=1}^{t} b_{i j}^{(k)}\left(X M_{k}\right) \\
&=X \sum_{k=1}^{t} b_{i j}^{(k)} M_{k} \quad(i, j=1, \ldots, p) .
\end{aligned}
$$

So if we let

$$
D_{i j}=\sum_{k=1}^{t} b_{i j}^{(k)} M_{k}, \quad(i, j=1, \ldots, p)
$$

then $D=\left(D_{i j}\right)$ is the matrix defined by (15) and we have, from (17), (18), and (19)

$$
C_{i j} X=X D_{i j} \quad(i, j=1, \ldots, p),
$$

so that the matrices $C$ and $D$ satisfy (3). Thus, by part 2 of theorem 2 , there exists a matrix $P$ such that

$$
P^{-1} C P=\left(\begin{array}{cc}
D & * \\
0 & E
\end{array}\right)
$$

so that $p r$ of the roots of $C$ are roots of $D$. Moreover, if the matrix $X$ is known, $D$ and $E$ can be found explicitly by using (11) to construct $P$.

\section{An Application to a Lattice Point Problem}

The example below is a matrix which arose in a problem involving lattice points at the National Bureau of Standards:

$$
\mathscr{A}=\left(\begin{array}{rrrrrrrrr}
4 & -1 & . & -1 & \beta & . & . & . & \cdot \\
-1 & 4 & -1 & -\beta & -1 & \beta & . & . & . \\
. & -1 & 4 & . & -\beta & -1 & . & . & . \\
-1 & -\beta & . & 4 & -1 & . & -1 & \beta & . \\
\beta & -1 & -\beta & -1 & 4 & -1 & -\beta & -1 & \beta \\
. & \beta & -1 & . & -1 & 4 & . & -\beta & -1 \\
. & . & . & -1 & -\beta & . & 4 & -1 & . \\
. & . & . & \beta & -1 & -\beta & -1 & 4 & -1 \\
. & . & . & . & \beta & -1 & . & -1 & 4
\end{array}\right) .
$$

If we partition into $3 \times 3$ submatrices we see

$$
\mathscr{A}=\left(\begin{array}{lll}
A & B & 0 \\
B^{T} & A & B \\
0 & B^{T} & A
\end{array}\right) .
$$

D. E. Rutherford [9] shows that a tridiagonal matrix,

$$
T=\left(\begin{array}{lllllllll}
a & b & & & & & & \\
b & a & b & & & & & \\
& b & a & b & & & & \\
& & & \cdot & & & & \\
& & & & \cdot & & & \\
& & & & & & & \\
& & & & & b & a & b \\
& & & & & & b & a
\end{array}\right)
$$

has characteristic roots which may be written in the form $\lambda_{k}=a+2 b \cos \theta_{k}$ where $\theta_{k}=k \pi / n+1, k=1, \ldots, n$.

The characteristic vector corresponding to $\lambda_{k}$ is

$$
x_{k}=\left(\sin \theta_{k}, \sin 2 \theta_{k}, \ldots, \sin n \theta_{k}\right) .
$$


The transformation for an i.r.p. tridiagonal matrix was given by the author in [6] and Rutherford also gives all the roots in the d.r.p. case.

The example (22) falls into none of the above categories, however, since $B \neq B^{T}$; but we show how the transformations discussed in section 4 can be used to simplify the matrix so that five of the roots may be found without having to solve an equation of degree higher than 2. I am grateful to D. E. Rutherford of the University of St. Andrews for giving me the other four roots.

We first use the transformation matrix, $P_{1}=(X \times I)$ where $X$ is the matrix of vectors (23) for a $3 \times 3$ tridiagonal matrix; i.e.,

$$
X=\frac{1}{2}\left(\begin{array}{rrr}
1 & \sqrt{2} & 1 \\
\sqrt{2} & 0 & -\sqrt{2} \\
1 & -\sqrt{2} & 1
\end{array}\right), X^{-1}=X,
$$

and $I$ is the identity matrix of order 3 . Then we find

$$
\mathscr{B}=P_{1}^{-1} \mathscr{A} P_{1}=\left(\begin{array}{ccc}
A-\sqrt{2} I & C^{T} & 0 \\
C & A & C^{T} \\
D & C & A+\sqrt{2} I
\end{array}\right),
$$

where

$$
C=\frac{B-B^{T}}{2}=\left(\begin{array}{rrr}
0 & \beta & 0 \\
-\beta & 0 & \beta \\
0 & -\beta & 0
\end{array}\right) \text {. }
$$

Since $A$ and also the matrices $A \pm \sqrt{2} I$ are all tridiagonal, we now use the transformation matrix $P_{2}=(I \times X)$ and find

$$
\mathscr{C}=P_{2}^{-1} B P_{2}=\left(\begin{array}{ccc}
D-\sqrt{2} I & C^{T} & 0 \\
C & D & C^{T} \\
0 & C & D+\sqrt{2} I
\end{array}\right),
$$

where

$$
\mathscr{D}=\operatorname{diag}(4-\sqrt{2}, 4,4+\sqrt{2}) .
$$

If we write the rows and columns of $\mathscr{C}$ in the order, 826435791, we obtain the matrix

$$
\mathrm{D}=\left(\begin{array}{ll}
E & 0 \\
* & F
\end{array}\right)
$$

with

$$
E=\left(\begin{array}{ll}
G & H \\
H & G
\end{array}\right), \quad F=\left(\begin{array}{cc}
K & L \\
L^{T} & M
\end{array}\right),
$$

where

$$
\begin{aligned}
& G=\left(\begin{array}{cc}
4+\sqrt{2} & \beta \\
\beta & 4-\sqrt{2}
\end{array}\right), H=-I_{2}, \\
& K=\left(\begin{array}{ccc}
4 & -\beta & . \\
-\beta & 4 & -\beta \\
\cdot & -\beta & 4
\end{array}\right), \quad L=\left(\begin{array}{ll}
0 & 0 \\
\beta & \beta \\
0 & 0
\end{array}\right),
\end{aligned}
$$

and $M=\operatorname{diag}(4+2 \sqrt{2}, 4-2 \sqrt{2})$. Thus $E$ is an o.r.p. circulant, so the roots of $E$ are those of $G+H$ and $G-H$, which are $4 \pm \beta \pm \sqrt{\beta^{2}+2}$.

Since $K$ is tridiagonal, let

$$
P_{3}=\left(\begin{array}{ll}
X & \\
& I_{2}
\end{array}\right) \text {. }
$$

Then

$$
P_{3}^{-1} F_{3}=\left(\begin{array}{ccccc}
4-\sqrt{2} \beta & . & . & \beta / \sqrt{2} & \beta / \sqrt{2} \\
\cdot & 4 & \cdot & \cdot & \cdot \\
\cdot & \cdot & 4+\sqrt{2} \beta & -\beta / \sqrt{2} & -\beta / \sqrt{2} \\
\beta / \sqrt{2} & . & -\beta / \sqrt{2} & 4+2 \sqrt{2} & . \\
\beta / \sqrt{2} & . & -\beta / \sqrt{2} & . & 4-2 \sqrt{2}
\end{array}\right) .
$$

Thus 4 is a root of this matrix, and when we delete the second row and column, we are left with a $4 \times 4$ matrix. I was unable to reduce this further by any relatively simple similarity transformation. However, D. E. Rutherford has found the other four roots of $F$ :

$$
4 \pm \sqrt{\beta^{2}+2 \beta+2} \pm \sqrt{\beta^{2}-2 \beta+2} .
$$

\section{References}

[1] S. N. Afriat, Composite matrices, Quart. J. Math., Oxford, 2d ser. 5, pp. 81-98 (1954).

[2] A. Brauer, Limits for the characteristic root of a matrix IV, Duke Math. J. 19, 75 (1952).

[3] B. Friedman, Eigenvalues of compound matrices, N.Y. Univ., Math. Research Group, Rept. No. TW-16 (1951).

[4] L. S. Goddard and H. Schneider, Matrices with a nonzero commutator, Proc. Cambridge Phil. Soc. 51, 551 (1955).

[5] E. Haynsworth, A reduction formula for partitioned matrices, J. Research NBS 64B, 171 (1960).

[6] E. Haynsworth, Applications of a theorem on partitioned matrices, J. Research NBS 63B, 73 (1959).

[7] C. C. MacDuffee, Theory of matrices (Chelsea Publ. Co., New York, N.Y., 1946).

[8] W. E. Roth, On direct product matrices, Bull. Am. Math. Soc. 40, 461 (1934).

[9] D. E. Rutherford, Some continuant determinants arising in physies and chemistry II, Proc. Roy. Soc. Edinburgh 63, 232 (1952).

[10] J. Williamson, The latent roots of a matrix of special type, Bull. Am. Math. Soc. 37, 585 (1931).

(Paper 65B1-41) 